\title{
Formando en ciudadanía a niñas y niños. Perspectiva feminista en los Planes de Formación Ciudadana (PFC) de las escuelas chilenas
}

\author{
Training citizenship for girls and boys. Feminist perspective \\ in the Citizen Formation Plans (PFC) of Chilean schools
}

\author{
Paula Subiabre Vergara ${ }^{a}$ \\ ${ }^{a}$ Facultad de Educación, Universidad de Barcelona. \\ paulasubiabre.v@gmail.com
}

\begin{abstract}
RESUMEN
La Ley 20911 que requirió la confección de Planes de Formación Ciudadana (PFC) ha permitido un avance al incorporar la ciudadanía a la experiencia escolar, pero se ha construido solo desde el punto de vista del ciudadano. La presente investigación tiene como objetivo analizar los PFC elaborados por veinte escuelas chilenas a partir de un enfoque feminista. En primer lugar, se analiza el modelo de ciudadanía desde el nivel de análisis feminista, lo que permite concluir que los PFC se han elaborado desde la perspectiva del ciudadano, y no han incluido las aportaciones del feminismo. En segundo lugar, se analiza cómo se incorpora esta perspectiva, identificando que las metodologías utilizadas son participativas y no cooperativas y los temas de debate que permitirían incorporar la perspectiva de género tienen escasa presencia. Por último, se plantean orientaciones para desarrollar una formación ciudadana con perspectiva feminista, con el objetivo de convertirse en una alternativa a la forma en que se está educando en ciudadanía.
\end{abstract}

Palabras clave: ciudadanía, género, feminismo, educación para la ciudadanía.

\begin{abstract}
Law 20911, which required the preparation of Citizen Training Plans, has allowed progress in incorporating citizenship into the school experience, but it has been built only from the citizen. The objective of this research is to analyze the PFCs prepared by twenty Chilean schools from a feminist perspective. First, the citizenship model is analyzed from a feminist level, which allows us to conclude that the PFCs have been elaborated from the citizen's perspective, and the contributions of feminism have not been included. Secondly, how the gender perspective is incorporated is analyzed, identifying methodologies used that are participatory and not cooperative, and subjects of discussion that will allow incorporating the gender perspective have little presence. Lastly, guidelines are presented to develop citizen training with a feminist perspective, to become an alternative to the way citizenship is being educated.
\end{abstract}

Key words: citizenship, gender, feminism, education to the citizenship. 
Estudios Pedagógicos XLVII N 3: 99-123, 2021

FORMANDO EN CIUDADANÍA A NIÑAS Y NIÑOS. PERSPECTIVA FEMINISTA EN LOS PLANES DE FORMACIÓN CIUDADANA (PFC) DE LAS ESCUELAS CHILENAS

\section{INTRODUCCIÓN}

Durante los últimos años se ha observado en Chile una creciente movilización feminista, que nos permite hablar de la existencia de una $4^{\mathrm{a}}$ ola. Estos movimientos se han caracterizado por tener una fuerte presencia en la calle y en los espacios educativos y han focalizado sus reivindicaciones en la educación "no sexista" y en la erradicación de todo tipo de violencia en función del género. Estas reivindicaciones son parte de un cambio transversal que pretende transformar la cultura patriarcal y el modelo económico capitalista en todos los aspectos de la realidad chilena. En este sentido, han cuestionado una ciudadanía androcéntrica y reproductiva del sistema social que tiende a perpetuar las desigualdades sociales y al mismo tiempo plantean la necesidad de pensar en nuevas formas de construir ciudadanía.

Teniendo en cuenta este contexto, resulta necesario desarrollar un enfoque feminista a la hora de discutir y reflexionar sobre cómo educar en ciudadanía incorporando la categoría de género. El género es una categoría de análisis que sostiene que las características que se le han atribuido a la mujer y al hombre no provienen de la naturaleza, sino que corresponden a construcciones culturales (Varela, 2018, p. 181). La exclusión de esta categoría implica concebir una ciudadanía universalista, es decir basada en un sujeto normativo hombre, de clase media, occidental, heterosexual, etc. que no toma en cuenta las particularidades y experiencias de los distintos colectivos sociales. En este sentido, la invisibilización de estas diferencias puede convertir a la educación ciudadana en un instrumento político que oculte estructuras de poder y desigualdades sociales (Arnot, 2009).

De esta manera entonces ¿Cómo estamos educando en ciudadanía? ¿La formación ciudadana en Chile tiene en cuenta la perspectiva feminista? Durante las últimas décadas ha existido una preocupación creciente por fomentar la educación para la ciudadanía en el sistema educativo chileno. En este contexto, el año 2016 se promulga la Ley 20.911 que demanda la creación de Planes de Formación Ciudadana (PFC) con el objetivo de brindar a "los estudiantes una preparación necesaria para asumir una vida responsable" y que busca "propender a la formación de ciudadanos, con valores y conocimientos para fomentar el desarrollo del país" (Ley 20911, 2016). ¿Qué sucede con la preparación para una vida responsable para las niñas y jóvenes? ¿Las niñas y jóvenes chilenas no serán ciudadanas que contribuyan al desarrollo del país?

En este artículo se exponen los resultados de una investigación empírica, que tuvo como objeto analizar los PFC diseñados por veinte escuelas chilenas entre el 2016 y 2019 a partir de un enfoque feminista, evaluando las posibilidades y limitaciones que ofrece la Ley 20911 para desarrollar una educación ciudadana con este enfoque.

Según Donoso (2018) para realizar un análisis de género existen dos niveles de acercamiento. El primero, denominado perspectiva de género, involucra una revisión de las desigualdades entre hombres y mujeres con el fin de visibilizarlas y repararlas. El segundo, denominado feminista, busca tomar una posición crítica frente al sistema patriarcal y al género como principio que estructura las jerarquías. Este nivel tiene como objetivo elaborar una nueva concepción de ciudadanía que incorpore valores, capacidades y experiencias de las mujeres y otros colectivos oprimidos; además de los aportes teóricos que los feminismos han realizado en la materia (Donoso, 2018).

Para el desarrollo de este artículo se utilizará el nivel de análisis feminista por lo que busca analizar cuál es el modelo de ciudadanía transmitido por los planes y si se incorporan los valores y aportes del feminismo a esa construcción. 
A los niveles de análisis de Donoso, se agregó un tercero que busca reflexionar sobre cómo se incorpora el enfoque feminista en los PFC. Para ello se identifican temáticas y metodologías que permiten trabajar esta perspectiva.

\section{MARCO INTERPRETATIVO PARA ANALIZAR LOS PFC DESDE LA MIRADA FEMINISTA}

\subsection{LA CIUDADANÍA Y LOS MODELOS DE DEMOCRACIA}

Según Cabrera (2002), la ciudadanía está compuesta de dos dimensiones: estatus y práctica. La ciudadanía como estatus corresponde al vínculo legal por el cual ciudadanos y ciudadanas poseen derechos y deberes por su pertenencia a una entidad jurídica. La ciudadanía como práctica es "una dimensión de naturaleza psicológica asociada a una identidad de ciudadano que le hace sentirse parte de una colectividad con la que se identifica y se reconoce" (p. 8).

La ciudadanía y su forma de ejercicio está en estrecha relación con el modelo de democracia imperante. Históricamente se han desarrollado tres modelos, democracia representativa, participativa y radical. El modelo de democracia representativa se desarrolló en Europa durante el siglo XX, dando origen al Estado de Bienestar, sistema que se basó en la redistribución de una serie de servicios sociales con carácter universal (Subirats y Parés, 2014). Una de sus características es la representatividad, es decir las autoridades son elegidas mediante el voto, y toman decisiones con respecto a la elaboración y ejecución de las políticas públicas que conciernen a quienes representan (Subirats y Parés, 2014).

El tipo de ciudadanía que se establece en este modelo de democracia es el de "ciudadano social" de Marshall. Según Aguiló (2009), este define la ciudadanía como un estatus que se le otorga a un individuo por el hecho de pertenecer a una comunidad, otorgándole derechos civiles, políticos y sociales. Este modelo ha sido denominado por Aguiló (2009) como ciudadanía de baja intensidad, en el que las personas se convierten en sujetos de derecho, pero con pocas posibilidades de ejercer su rol ciudadano, es decir "significa ser un obediente ciudadano-siervo que queda reducido a la condición periódica de elector sin el poder sustantivo necesario para participar en los procesos de toma de decisiones" (p. 16).

La democracia participativa o de gobernanza, surge por la necesidad de responder a las críticas del modelo representativo, especialmente en relación con su falta de eficiencia en la generación de políticas públicas y la falta de legitimidad. La democracia participativa propone un ejercicio político más igualitario, en el cual las personas se involucran en la elaboración de las políticas públicas. En este modelo, la representación es complementada con estrategias de participación ciudadana (Subirats y Parés, 2014).

El tipo de ciudadanía que se persigue es mucho más activo, ya que al estatus otorgado se le incorporan prácticas participativas, aumentando con ello su sentido de pertenencia. Junto con esto, ciudadanas y ciudadanos pueden deliberar sobre ciertas temáticas que les competen. Sin embargo, los espacios, temas y personas que participan no son espontáneos ni decididos por la ciudadanía, sino que vienen determinados por el Estado.

En el escenario actual de crisis de representatividad (PNUD, 2015), surgen cada vez más iniciativas ciudadanas asociadas a los movimientos sociales, que prefieren formas de autoorganización independientes de las políticas estatales. Estas experiencias se relacionan 
con el modelo de democracia radical que plantea que la búsqueda de consenso propia de las democracias liberales excluye a las personas diferentes (Parés y Subirats, 2014). Sus ideas centrales son el respeto a la diversidad, la colaboración, la conectividad, la presión y la implementación. En la práctica, esto se traduce en mecanismos de participación que se producen de manera comunitaria, generalmente al margen del Estado, que buscan dar solución a problemas colectivos (Subirats y Parés, 2014). Este modelo implica la construcción de ciudadanías de alta intensidad, donde la ciudadanía, además de ser un estatus es una práctica social colectiva, solidaria y participativa que garantiza las condiciones para ejercicio efectivo de los derechos (Aguiló, 2009).

Este diagnóstico es coincidente con el proceso de politización que, de acuerdo al informe del PNUD (2015), atraviesa la sociedad chilena en las últimas décadas. Este se define por una ampliación del debate público, el aumento de la conflictividad y el involucramiento ciudadano, teniendo como características principales la creciente importancia de los movimientos sociales en el debate público y la amplia demanda ciudadana por cambios profundos en diferentes ámbitos de la sociedad chilena.

\subsection{FEMINISMO Y CIUDADANÍA}

El feminismo es un movimiento social y político que tiene sus raíces en el siglo XVIII y se constituye al mismo tiempo como movimiento político-social y como teoría. Según Nuria Varela (2018), el feminismo no solo busca la igualdad de condiciones para las mujeres, sino que cuestiona el orden social con el objetivo de transformar la realidad.

En estos doscientos años de existencia del feminismo, han existido diferentes corrientes, sin embargo, comparten elementos. En primer lugar, entienden que todas las personas son igualmente diferentes, es decir que se debe respetar la individualidad y valorar la diversidad. También defienden que todas las formas de discriminación y opresión son condenables, por lo que los feminismos exigen no solo más derechos para las mujeres, sino una transformación de las estructuras sociales, que apunten a la desaparición de la opresión y desigualdades sociales (Facio, 2005). Por último, buscan relevar que lo personal es político, es decir que existe una relación entre la experiencia personal y privada de las mujeres y las estructuras de poder (Hanish, 2016).

El género es una de las principales categorías de análisis del feminismo. Se refiere a que los rasgos que se espera que tengan mujeres y hombres, no provienen de la biología, sino que son construcciones culturales. En este sentido, estas desigualdades tienen un carácter social y cultural y la construcción de las identidades se desarrolla a partir de la asignación de características, roles y responsabilidades por la pertenencia al sexo biológico (Abasolo y Montero, 2012).

Entre las características del género, se destaca que es una categoría vinculante, es decir que lo masculino y lo femenino están en una relación de complementariedad y dependencia (Sau, 1990). Los géneros son además jerarquizados, posicionándose el masculino como dominante y el femenino como subordinado, mientras que quienes no se identifican con estas categorías quedan excluidos de lo social. Por lo tanto, el género establece una relación jerárquica y de poder.

Al definir que la opresión y dominación de la mujer no está dada por condiciones naturales, las feministas han podido desarrollar alternativas políticas para luchar por la transformación social. En los primeros años, la mujer se entendió como sujeto social que 
tenía en común la opresión derivada de su posición estructural en la sociedad. A partir de los años 80s se comienza a cuestionar esta identidad homogénea y fuerte, y empieza a ser reemplazada por la visión de una identidad basada en la diversidad de experiencias. Esto se debió a que, en EEUU, mujeres negras y latinas se dieron cuenta de que el discurso de las feministas (mujeres blancas de clase media), no las representaba (Stolcke, 2004). A partir de ahí, se desarrolla un debate que pone en tensión la necesidad de preservar la unidad de las mujeres y al mismo tiempo poner énfasis en su diversidad. Comienza a sustituirse la idea de Mujer por la de Mujeres, haciendo hincapié en los diferentes tipos de opresión que existen en las experiencias de la diversidad de mujeres, además de la importancia de comprender el género en relación, interconexión, reciprocidad con otros sistemas de opresión como clase, etnia, orientación sexual, entre otras, aspecto que han enfatizado las perspectivas interseccionales (Stolcke, 2004; Troncoso et al., 2019).

Por su parte Monique Wittig (como se citó en Burgos, 2010) cuestiona el carácter natural no solo del género, sino también del sexo. En ese sentido, la categoría mujer y su correspondiente heterosexualidad son categorías inventadas para oprimir y subordinar a la mujer. Para Wittig existe una construcción social no solo en la subordinación de las mujeres, sino en la división de la humanidad entre hombres y mujeres. Sostiene su interpretación mediante la figura de la lesbiana, que pone en evidencia la artificialidad de las marcas sexuales, puesto que "nos aventura por el camino de una sexualidad no heterocentrada, no genitalizada; una sexualidad que erotiza el cuerpo en toda su extensión y pluralidad" (Wittig, como se citó en Burgos, 2010). Desde su lectura el fin de la opresión y subordinación estaría dado por la superación de los géneros.

Otro de los conceptos fundamentales de la teoría feminista es el patriarcado, que corresponde a una forma de organización política, económica y social basada en la autoridad del hombre sobre las mujeres y los niños y niñas en la familia, en el que su dominio se proyecta a toda la sociedad. Según Rosa Cobo (1995), el patriarcado es un sistema de pactos interclasistas entre hombres permanente en el tiempo, que se sostiene gracias a la coerción y el convencimiento.

El sexismo, por su parte, corresponde al conjunto de métodos utilizados por el sistema patriarcal para continuar con la condición de inferioridad y subordinación de la mujer y personas no binarias. Está presente en todos los espacios y ámbitos de la vida. Se puede apreciar en la división sexual del trabajo, en la violencia de género, en el trabajo doméstico, la diferencia entre las carreras, la doble jornada de trabajo, entre otros (Sau, 1990).

Las reflexiones que han realizado las teóricas feministas sobre ciudadanía surgen de la crítica a la teoría política tradicional. Carol Pateman (1988), denunció la contradicción que existe entre una igualdad formal de las mujeres y su subordinación social y sometimiento como esposas. Esta idea tiene su origen en el contrato social. Para la autora, este contrato es un pacto sexual-social entre hombres, en el que se excluye a las mujeres y se las relega al ámbito privado, estableciéndose una dicotomía entre lo público y lo privado (masculinofemenino).

Arnot (2009), tomando como referencia a Pateman, señala que la separación entre lo público y lo privado tiene consecuencias en la conformación de la ciudadanía. Los hombres al estar asociados al dominio público "se transforman en seres sociales y públicos, mientras que las mujeres a quienes se interpreta como símbolo del dominio privado quedan por definición, excluidas del orden social" (p. 51). Por lo tanto, a las mujeres se les niega la posesión de poder político y la capacidad de agencia. 
El feminismo de la diferencia sostiene que la teoría política moderna ha basado su pensamiento en la idea de la universalidad. Sin embargo, ese universalismo es un constructo que se ha concebido desde la particularidad del hombre. El feminismo ha contribuido a reformular los paradigmas de la teoría política actual al afirmar que las mujeres podían obtener la igualdad social solo en la medida que se les reconociera la diferencia (Ochman, 2006). Iris Young (1990), propone la construcción de una ciudadanía diferenciada. Una política basada en la diferencia de grupo implica un nuevo concepto de igualdad. En el ideal universalizador, la igualdad consiste en tratar a todas las personas según los mismos principios, en cambio la política de la diferencia sostiene que la inclusión y participación de todas las personas implica un tratamiento diferente para los grupos oprimidos.

Chantal Mouffe (1999), comparte la crítica que realiza el feminismo de la diferencia a la idea de la universalidad, pero también le reprocha que posea una visión esencialista del género que limita la construcción de una democracia radical. Para desarrollar este proyecto se debe descartar la idea de un sujeto racional y transparente heredado de la modernidad, ya que un individuo puede a la vez ser dominante en una relación y subordinado en otra. Para el proyecto de democracia radical se concibe la identidad de los sujetos como algo múltiple y contradictorio. Para Mouffe (1999), esta idea de sujeto es la que permite establecer una nueva ciudadanía, entendida no como una única identidad, sino como "un principio articulador que afecta a las diferentes posiciones subjetivas del agente social, aunque reconociendo una pluralidad de lealtades específicas y el respeto de la libertad individual" (p. 101). Esta nueva concepción de ciudadanía es la que permite llevar al proyecto de democracia radical, cuyo objetivo es profundizar la revolución democrática y conectar las distintas luchas que existen en la actualidad.

\subsection{EDUCACIÓN CIUDADANA E INCLUSIÓN DE LA PERSPECTIVA FEMINISTA}

La educación para la ciudadanía corresponde a los conocimientos, habilidades y valores que transmite la escuela, con el objetivo de formar a las y los jóvenes para participar de forma activa en la democracia, de modo que "se preparen para asumir sus roles y sus responsabilidades como ciudadanos y ciudadanas de una sociedad libre, plural y tolerante" (Pagès y Santisteban, 2007, p. 3).

Westheimer y Khane (2002) analizaron 10 programas de educación ciudadana en el mundo anglosajón, a partir de los que establecieron tres concepciones de "buen" ciudadano/a. El primer tipo, "ciudadanía personalmente responsable", corresponde a programas en que se destacan valores como la honestidad, la integridad, el respeto, la autodisciplina y el trabajo duro. Una persona característica de este modelo es quien trabaja, paga impuestos, obedece leyes y contribuye en situaciones de catástrofe. Según los autores, en este modelo existe un énfasis en la visión individualista del/de la buen/a ciudadano/a.

La "ciudadanía participativa", se caracteriza por enfocarse en la preparación del estudiantado en la participación activa. En estos programas, la concepción del/ de la buen/a ciudadano/a está asociado a personas que participan activamente en asuntos públicos y de su comunidad local. Desarrolla valores como entendimiento común, confianza, compromiso colectivo. Estos programas buscan un mayor compromiso social en tareas colectivas, destacando acciones para ayudar a los más necesitados. Sin embargo, evitan la crítica sociopolítica y no buscan la transformación social (Westheimer y Khane, 2002). 
La "ciudadanía orientada a la justicia" se destaca por estar encaminada a develar las injusticias y a profundizar el sistema democrático. El/la buen/a ciudadano/a estaría representado por el/la activista. Los programas se caracterizan por su énfasis en la evaluación crítica de las estructuras sociales, políticas y económicas, considera estrategias colectivas para transformar las injusticias (Westheimer y Khane, 2002).

Para los autores, el énfasis individual que posee el modelo de ciudadanía responsable es inadecuado para los desafíos que presenta educar en una ciudadanía democrática, ya que oscurece la necesidad de iniciativas colectivas y obvia el análisis de las causas sociales de los problemas actuales (Westheimer y Khane, 2002). Por otra parte, el modelo de ciudadanía orientada a la justicia pareciera ser el más propicio para desarrollar una ciudadanía con enfoque feminista, debido a que se centra en la crítica y la transformación social necesarias para romper con las estructuras patriarcales.

La escuela -espacio primordial de formación de ciudadanas y ciudadanos- es una de las instituciones que juegan un papel fundamental en la reproducción del sistema patriarcal expresado a través del currículum manifiesto y del currículum oculto (Guerrero et al., 2011). De ahí la necesidad de transversalizar la perspectiva de género o fomentar la coeducación, es decir, desarrollar acciones en todas las áreas y niveles de enseñanza que incorporen las preocupaciones y experiencias y aportaciones tanto de las mujeres como de los hombres, así como la revisión de situaciones de perpetuación de roles o estereotipos de género (Guerrero et al., 2011). Para Sánchez e Iglesias (2008), no adoptar de forma intencionada la perspectiva de género en la escuela, limitará la capacidad de imaginar una organización social diferente.

La incorporación de la perspectiva de género en la educación también ha tenido repercusiones en la educación para la ciudadanía. Para Rosa Cobo (2008), la educación para la ciudadanía en perspectiva feminista debe enfatizar en la revisión crítica de los sistemas democráticos actuales, reconociendo los déficits existentes para ciertos sectores de la sociedad.

Para Abasolo y Montero (2012), el estudiantado debe analizar y reformular el concepto de igualdad. En la teoría política, la igualdad como concepto moderno y occidental se ha construido desde un punto de vista universalista. Sin embargo, encierra la exclusión de determinados colectivos sociales. Se debe avanzar, por lo tanto, hacia una igualdad en diversidad.

De acuerdo a Arnot (2009), se debe incorporar el concepto de ciudadanía sexual y reproductiva. Este concepto se refiere al reconocimiento del control que han hecho los hombres de la capacidad reproductiva de las mujeres y la demanda por nuevos derechos relacionados con la sexualidad y la reproducción. Según Arnot (2009), este concepto permite incluir en el debate aspectos como el derecho a la autodeterminación sexual y reproductiva, el reconocimiento de la diversidad sexual, el derecho a cada persona a elegir sus parejas sexuales, el respeto a la expresión sexual, entre otros temas.

Una educación ciudadana con enfoque feminista también debe reflexionar en torno a la metodología que se utiliza para enseñar. La pedagógica tradicional, según Roset et al. (2008), se basa en una concepción androcéntrica del conocimiento. Esto se traduce en una metodología tradicional, donde el profesorado es la fuente de conocimiento, mientras los y las alumnas son meros receptores de este. Esta forma de educar no propicia la inclusión de la diversidad de estudiantes. La escuela coeducadora ${ }^{1}$, en cambio, debe

La coeducación es un modelo de escuela que integra los valores, experiencias, y aportaciones de todas las personas, independiente de su sexo. Tiene especial atención en denunciar y corregir perpetuación de roles y estereotipos sexistas (Sánchez e Iglesias, 2008). 
tener una práctica docente transformadora, definida "por su inclusividad, por el respeto a la diferencia, por el intento de compensar las desigualdades sociales y por una concepción de saberes no estereotipada" (p. 86). Para ello, una de las estrategias fundamentales es el trabajo cooperativo, ya que la participación en pequeños grupos permite desarrollar competencias de colaboración, expresar las ideas de todos/as en igualdad de condiciones, facilita la reflexión, la participación, etc. (Roset et al., 2008).

\section{METODOLOGÍA}

El estudio se circunscribe al paradigma cualitativo orientado a la comprensión, con el objeto de revisar el estado actual de la educación ciudadana en Chile. Se efectuó a través del análisis documental, que se basa en el examen de documentos generados por las instituciones educativas (Corbetta, 2007). Los PFC corresponden al producto material en el que se plasman las ideas educativas que declara trabajar cada establecimiento educativo.

La muestra se compuso de veinte PFC, escogidos mediante un muestreo intencional de acuerdo a criterios establecidos a priori (Sabariego, 2016). Los criterios se establecieron según los indicadores de Desarrollo Personal y Social de la evaluación SIMCE². Finalmente, se utilizaron planes que estuvieran publicados y que incluyeran los siguientes apartados: objetivos, fundamentación y acciones a realizar. A partir de estos criterios se configuró la muestra que puede apreciarse en la Tabla 1.

Tabla 1. Características de la muestra utilizada

\begin{tabular}{|l|l|l|l|l|l|l|}
\hline & $\begin{array}{c}\text { Mixta o } \\
\text { segregada }\end{array}$ & $\begin{array}{c}\text { Confesión } \\
\text { religiosa }\end{array}$ & \multicolumn{1}{|c|}{ Dependencia } & $\begin{array}{c}\text { Grupo } \\
\text { socioeconómico } \\
\text { GSE }\end{array}$ & $\begin{array}{c}\text { Indicador de } \\
\text { participación } \\
\text { ciudadana }\end{array}$ & $\begin{array}{c}\text { Indicador } \\
\text { género }\end{array}$ \\
\hline 1 & Mixta & Laico & Part. Subvencionado & Medio alto & 74 similar GSE & similar \\
\hline 2 & Mujeres & Laico & Municipal & Medio alto & 76 similar GSE & - \\
\hline 3 & Mixta & Laico & Municipal & Medio & 73 similar GSE & similar \\
\hline 4 & Mixta & Religiosa & Part. Subvencionado & Medio & 83 más alto GSE & similar \\
\hline 5 & Mixta & Religiosa & Part. Subvencionado & Medio bajo & 75 similar GSE & similar \\
\hline 6 & Mixta & Laico & Municipal & Medio alto & 74 similar GSE & similar \\
\hline
\end{tabular}

2 El SIMCE (Sistema de Medición de la Calidad de la Educación) corresponde a una evaluación estandarizada que tiene como objeto evaluar los aprendizajes alcanzados por cada establecimiento chileno. Además de las pruebas se recoge información a través de cuestionarios aplicados a estudiantes, docentes y apoderados. El indicador Participación Ciudadana mide las percepciones y actitudes frente a la participación del establecimiento educativo, el compromiso de los miembros de la comunidad educativa y la promoción de la vida democrática (Agencia de Calidad de Educación, s/f). De acuerdo con este indicador se escogieron escuelas que tuvieran un índice superior a 70 (medición entre 0 y 100) y que además estuviesen en un nivel similar o superior con respecto a su mismo grupo socioeconómico. El indicador de Equidad de Género evalúa el logro equitativo de resultados de aprendizaje obtenido por mujeres y hombres en las pruebas de Lenguaje y Matemáticas. Se determina observando las diferencias existentes en sus resultados (Agencia de Calidad de Educación, s/f). Se escogieron establecimientos que tuvieran resultados similares. 
Estudios Pedagógicos XLVII N 3: 99-123, 2021 FORMANDO EN CIUDADANÍA A NIÑAS Y NIÑOS. PERSPECTIVA FEMINISTA EN LOS PLANES DE FORMACIÓN CIUDADANA (PFC) DE LAS ESCUELAS CHILENAS

\begin{tabular}{|c|l|l|l|l|l|l|}
\hline 7 & Mixta & Religiosa & Part. Pagado & Alto & 75 similar GSE & similar \\
\hline 8 & Mixta & Religiosa & Part. Subvencionado & Alto & 77 similar GSE & similar \\
\hline 9 & Mujeres & Religiosa & Part. Subvencionado & Medio bajo & 82 más alto GSE & - \\
\hline 10 & Mixta & Laico & Part. Subvencionado & Medio & 82 similar GSE & similar \\
\hline 11 & Mixta & Laico & Part. Subvencionado & Medio & 79 similar GSE & Similar \\
\hline 12 & Mixta & Religiosa & Part. Subvencionado & Medio & 80 similar GSE & similar \\
\hline 13 & Mixta & Laico & Part. Subvencionado & Medio bajo & 79 similar GSE & similar \\
\hline 14 & Mixta & Religiosa & Part. Pagado & Medio alto & 80 similar GSE & similar \\
\hline 15 & Mixta & Laico & Municipal & Medio bajo & 80 similar GSE & similar \\
\hline 16 & Mixta & Religiosa & Part. Subvencionado & Medio & 79 similar GSE & similar \\
\hline 17 & Mixta & Laico & Municipal & Medio bajo & 81 similar GSE & similar \\
\hline 18 & Mixta & Religiosa & Part. Subvencionado & Bajo & 84 más alto GSE & similar \\
\hline 19 & Mixta & Laico & Part. Subvencionado & Medio & 83 más alto GSE & similar \\
\hline 20 & Mixta & Laico & Part. Subvencionado & Bajo & 80 similar GSE & similar \\
\hline
\end{tabular}

Fuente: elaboración propia a partir de datos SIMCE $2018 .^{3}$

Para su comprensión y posterior interpretación se realizó un análisis de contenido, que corresponde a la revisión sistemática de un texto "para lo cual identifica unidades de análisis que clasifica siguiendo un proceso de categorización y cuya interpretación posterior permite resumir el contenido manifiesto" (Cabrera, 2011, p. 121). Para la categorización se utilizó en primer lugar un sistema inductivo que surgió del marco teórico y posteriormente, se incluyeron nuevas categorías y subcategorías que surgieron del análisis de los planes, como se pueden apreciar en la tabla 2.

\footnotetext{
En el sistema educativo chileno existen tres tipos de establecimientos según su dependencia administrativa. Los establecimientos municipales son financiados y gestionados por una entidad pública y tienen un carácter gratuito. Los establecimientos particulares subvencionados se financian con aportes estatales, además del pago de una mensualidad por parte de las familias y son gestionados por una entidad privada. Los establecimientos particulares pagados se gestionan por entidades privadas y son financiados a través de la mensualidad que pagan las familias.
} 
Tabla 2. Categorías y subcategorías del análisis de contenido

\begin{tabular}{|c|c|c|c|}
\hline \multicolumn{2}{|c|}{ Categorías } & \multicolumn{2}{|r|}{ Subcategorías } \\
\hline \multirow[t]{3}{*}{ Ciudadanía } & \multirow{3}{*}{$\begin{array}{l}\text { Estatus por el } \\
\text { que se } \\
\text { otorgan } \\
\text { derechos a las } \\
\text { personas y } \\
\text { capacidad de } \\
\text { participar en } \\
\text { el espacio } \\
\text { público. }\end{array}$} & $\begin{array}{l}\text { Ciudadanía } \\
\text { responsable }\end{array}$ & $\begin{array}{l}\text { Las y los ciudadanos conocen y respetan la } \\
\text { institucionalidad política. Actúan } \\
\text { responsablemente en su comunidad y } \\
\text { participan del sistema democrático a través } \\
\text { de la elección de sus representantes. Se } \\
\text { asocia a una ciudadanía de baja intensidad. }\end{array}$ \\
\hline & & $\begin{array}{l}\text { Ciudadanía } \\
\text { participativa }\end{array}$ & $\begin{array}{l}\text { Las y los ciudadanas participan activamente } \\
\text { en su comunidad. Hay un compromiso social } \\
\text { e involucramiento en tareas colectivas, } \\
\text { especialmente con las personas que lo } \\
\text { necesitan. No existe la crítica política. }\end{array}$ \\
\hline & & $\begin{array}{l}\text { Ciudadanía } \\
\text { orientada a la } \\
\text { justicia }\end{array}$ & $\begin{array}{l}\text { Está encaminada a develar las injusticias y a } \\
\text { promover una profundización del sistema } \\
\text { democrático. Las y los ciudadanos evalúan } \\
\text { críticamente las estructuras sociales, } \\
\text { políticas, económicas y culturales y buscan la } \\
\text { transformación social. }\end{array}$ \\
\hline \multirow[t]{2}{*}{ Igualdad } & \multirow{2}{*}{$\begin{array}{l}\text { Principio que } \\
\text { establece que } \\
\text { todos los } \\
\text { seres } \\
\text { humanos son } \\
\text { iguales. }\end{array}$} & $\begin{array}{l}\text { Igualdad de } \\
\text { Derechos }\end{array}$ & $\begin{array}{l}\text { Condición propia de los seres humanos, en la } \\
\text { que todas las personas tienen los mismos } \\
\text { derechos y oportunidades de desarrollo en } \\
\text { todos los ámbitos. }\end{array}$ \\
\hline & & $\begin{array}{l}\text { Igualdad en } \\
\text { diversidad }\end{array}$ & $\begin{array}{l}\text { La igualdad desde la perspectiva feminista } \\
\text { debe provenir del reconocimiento de la } \\
\text { diferencia. Es decir, requiere un tratamiento } \\
\text { diferente para los grupos oprimidos y } \\
\text { desventajados. }\end{array}$ \\
\hline \multirow[t]{3}{*}{ Valores } & \multirow{3}{*}{$\begin{array}{l}\text { Son } \\
\text { principios o } \\
\text { cualidades } \\
\text { que posee una } \\
\text { persona o } \\
\text { grupo de } \\
\text { personas. }\end{array}$} & $\begin{array}{l}\text { Valores del } \\
\text { modelo de } \\
\text { coeducación }\end{array}$ & $\begin{array}{l}\text { Corresponden a valores que permiten la } \\
\text { inclusión de todos y todas las estudiantes } \\
\text { como la diversidad, la empatía, la } \\
\text { cooperación. }\end{array}$ \\
\hline & & $\begin{array}{l}\text { Valores } \\
\text { tradicionalmente } \\
\text { asociados a lo } \\
\text { femenino }\end{array}$ & $\begin{array}{l}\text { Se busca dar valor a aquellos } \\
\text { tradicionalmente asociados al ámbito } \\
\text { femenino/privado como la ternura, el amor, } \\
\text { el cuidado. }\end{array}$ \\
\hline & & $\begin{array}{l}\text { Valores que } \\
\text { permiten luchar } \\
\text { contra la } \\
\text { discriminación }\end{array}$ & $\begin{array}{l}\text { Son aquellos que permiten luchar contra la } \\
\text { discriminación como la justicia y la no } \\
\text { discriminación. }\end{array}$ \\
\hline
\end{tabular}




\begin{tabular}{|c|c|c|c|}
\hline \multirow[t]{3}{*}{ Metodologías } & \multirow[t]{3}{*}{$\begin{array}{l}\text { Conjunto de } \\
\text { técnicas de } \\
\text { enseñanza } \\
\text { articuladas } \\
\text { entre sí que } \\
\text { se dirigen a la } \\
\text { consecución } \\
\text { de objetivos. }\end{array}$} & $\begin{array}{l}\text { Metodologías } \\
\text { tradicionales }\end{array}$ & $\begin{array}{l}\text { Estrategias de enseñanza que ponen el acento } \\
\text { en lo que el profesorado enseña. El } \\
\text { estudiantado es receptor. }\end{array}$ \\
\hline & & $\begin{array}{l}\text { Metodologías } \\
\text { participativas no } \\
\text { cooperativas }\end{array}$ & $\begin{array}{l}\text { Técnicas de enseñanza aprendizaje que } \\
\text { involucran la participación del estudiantado } \\
\text { de manera individual. }\end{array}$ \\
\hline & & $\begin{array}{l}\text { Metodologías } \\
\text { participativas } \\
\text { cooperativas }\end{array}$ & $\begin{array}{l}\text { Las/los estudiantes trabajen en colaboración } \\
\text { permitiendo el aprendizaje de todos/as los/las } \\
\text { integrantes del grupo. }\end{array}$ \\
\hline \multirow[t]{7}{*}{$\begin{array}{l}\text { Temáticas que } \\
\text { permiten } \\
\text { incorporar la } \\
\text { perspectiva } \\
\text { feminista }\end{array}$} & \multirow{7}{*}{$\begin{array}{l}\text { Corresponden } \\
\text { a temas de } \\
\text { debate actual } \\
\text { que ha } \\
\text { relevado el } \\
\text { movimiento } \\
\text { feminista y } \\
\text { que permiten } \\
\text { incorporar } \\
\text { esta } \\
\text { perspectiva al } \\
\text { trabajo sobre } \\
\text { ciudadanía. }\end{array}$} & $\begin{array}{l}\text { Derechos de las } \\
\text { mujeres }\end{array}$ & $\begin{array}{l}\text { Las mujeres y niñas deben tener igualdad de } \\
\text { derechos y vivir libres de discriminación. } \\
\text { Además, se refiere a derechos específicos que } \\
\text { se le conceden a las mujeres y a las niñas. }\end{array}$ \\
\hline & & $\begin{array}{l}\text { Violencia de } \\
\text { género }\end{array}$ & $\begin{array}{l}\text { Corresponde a cualquier acto violento o } \\
\text { agresión ejercida hacia las mujeres y } \\
\text { disidencias por razones de género. }\end{array}$ \\
\hline & & $\begin{array}{l}\text { Aportes del } \\
\text { feminismo }\end{array}$ & $\begin{array}{l}\text { Explicitar los aportes que ha desarrollado el } \\
\text { movimiento y teoría feminista al desarrollo } \\
\text { de la política actual. }\end{array}$ \\
\hline & & $\begin{array}{l}\text { Diversidad sexual } \\
\text { y de género }\end{array}$ & $\begin{array}{l}\text { Respeto de la diversidad de sexos, } \\
\text { orientaciones sexuales e identidades de } \\
\text { género. }\end{array}$ \\
\hline & & $\begin{array}{l}\text { Discriminación de } \\
\text { género }\end{array}$ & $\begin{array}{l}\text { Trato desigual hacia una persona o colectivo } \\
\text { debido a su sexo u orientación sexual. }\end{array}$ \\
\hline & & Interseccionalidad & $\begin{array}{l}\text { Fenómeno por el cual las personas sufren una } \\
\text { situación de opresión debido a su pertenencia } \\
\text { a diferentes categorías como género, etnia, } \\
\text { clase y orientación sexual. }\end{array}$ \\
\hline & & $\begin{array}{l}\text { Estereotipos de } \\
\text { género }\end{array}$ & $\begin{array}{l}\text { Se incorpora el tema a la reflexión en la } \\
\text { escuela con el objetivo de superar los } \\
\text { estereotipos de género. }\end{array}$ \\
\hline
\end{tabular}

Fuente: Elaboración propia a partir de la revisión bibliográfica realizada. 


\section{RESULTADOS}

\subsection{MODELO DE CIUDADANÍA}

Este apartado está relacionado con el nivel de análisis denominado feminista, en el que se busca describir el modelo de ciudadanía que está detrás de los PFC, y contrastarlo con los principios de una ciudadanía con enfoque feminista. Para ello se revisaron las categorías de análisis: tipo de ciudadanía, visión de igualdad y valores.

\subsubsection{Tipo de ciudadanía}

El currículum ha reemplazado una formación ciudadana basada en la alfabetización política, por una en que los y las estudiantes "vivan" la experiencia de ser ciudadanos/as. Sin embargo, como se puede apreciar en la figura 1, el análisis de los PFC da cuenta de una alta presencia del modelo de ciudadanía responsable. Se entiende por este tipo de ciudadanía "la facultad que posee una persona dentro de un Estado para participar responsablemente en la vida política del mismo, eligiendo y siendo elegido" (Escuela 1). Este tipo de ciudadanía se traduce en acciones como: la elección de representantes, la participación en actos cívicos, celebración de efemérides, el conocimiento de la institucionalidad política, etc.

Figura 1. Gráfico tipos de ciudadanía.

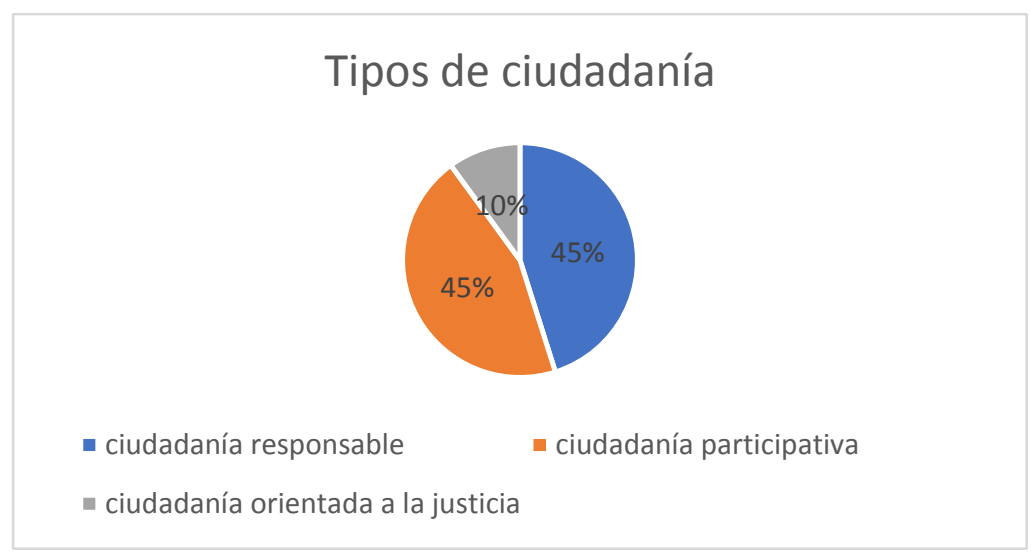

Fuente: Elaboración propia a partir del análisis de los PFC escogidos.

También tiene relevancia la subcategoría ciudadanía participativa. Este tipo de ciudadanía tiene el objetivo de "contribuir al desarrollo de una cultura democrática y de participación activa, en la que todos los integrantes de la comunidad educativa cuenten con la posibilidad de ser escuchados y sean propositivos en relación con el quehacer de la escuela" (Escuela ${ }^{\circ} 2$ ). Esto se traduce en acciones como "el desarrollo de proyectos por grupos de trabajo, que permita resolver problemáticas de la comunidad o que sean de 
interés público" (Escuela n²). Este tipo de actividades fomentan una ciudadanía activa, pero sin crítica al sistema social y político imperante.

La categoría ciudadanía orientada a la justicia tiene escaso desarrollo en los PFC analizados. Este tipo de ciudadanía la conforman "hombres y mujeres conscientes, capaces de trascender, agentes de cambio y protagonistas de su circunstancia" (Escuela $n^{\circ} 7$ ). Es decir, una ciudadanía que apunta a la transformación social. Esto se traduce en ciudadanas/ os que sean capaces por ejemplo de "hacer una evaluación crítica al modelo neoliberal imperante en nuestro país reconociendo la implicancia en su vida personal y social" (Escuela $\mathrm{n}^{\circ}$ 7). Además de la escasa presencia que se le da a este tipo de ciudadanía, no se desarrollan acciones para fomentarla. Por tanto, se puede inferir, que corresponde más a una declaración de principios, que al ejercicio cotidiano del estudiantado.

Figura 2: Gráfico tipo de ciudadanía según grupo socioeconómico.

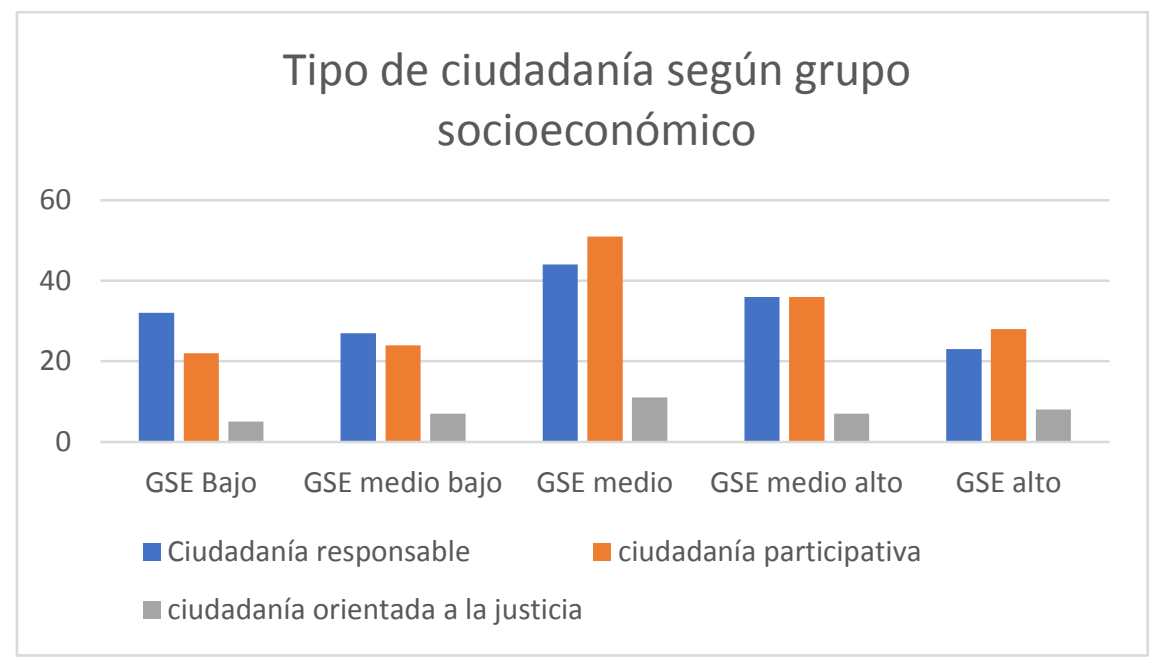

Fuente: Elaboración propia a partir del análisis de los PFC escogidos.

Si se observa la tendencia de los tipos de ciudadanía de las veinte escuelas analizadas según grupo socioeconómico (figura 2), se puede apreciar que en ninguno de los grupos se le da relevancia a la ciudadanía orientada a la justicia. Sí pareciera haber un mayor desarrollo de la ciudadanía participativa por sobre la ciudadanía responsable a medida que aumenta la condición socioeconómica de los establecimientos. Desgraciadamente en los grupos bajo y medio bajo, es donde mayormente se desarrolla una ciudadanía de baja intensidad, lo que resulta preocupante, pues la formación ciudadana corre el riesgo de convertirse en una mera reproducción del sistema imperante.

Al observar estos resultados desde una mirada feminista, se puede afirmar que los veinte PFC analizados aportan escasas posibilidades para fomentar un modelo de ciudadanía que rompa con el sistema patriarcal. En primer lugar, el modelo de ciudadanía debiese permitir 
la inclusión de todos los colectivos sociales y para ello se debe fomentar la participación. La subcategoría ciudadanía participativa tiene una presencia importante, pero no sobrepasa a la de ciudadanía responsable. Junto con esto, la primera es mayor en los grupos socioeconómicos medios y altos, por lo que existen más posibilidades de participar para los niños y niñas que provienen de familias con mejores condiciones socioeconómicas.

Además, se debe desarrollar una ciudadanía orientada a la justicia, pues es el pensamiento crítico el que le permite al estudiantado denunciar y transformar el sexismo y las estructuras patriarcales aun existentes en las sociedades contemporáneas.

\subsubsection{Igualdad}

La igualdad es uno de los principios centrales de una ciudadanía con enfoque feminista. Lamentablemente, tiene escaso desarrollo en los PFC. De hecho, cinco de los veinte PFC analizados ni siquiera lo mencionan y no existen en los planes acciones concretas en el que se desarrolle este principio.

Como se puede apreciar en la figura 3, la mayoría de los PFC conciben este principio como igualdad de derechos, por ejemplo: "respetar y defender la igualdad de derechos esenciales de todas las personas, sin distinción de sexo, edad, condición, física, etnia, religión o situación económica" (Escuela $\left.\mathrm{n}^{\circ} 19\right)$. Es decir, tienen una concepción tradicional y universal del concepto de igualdad. El problema de esta visión es que invisibiliza las desigualdades estructurales y desconoce que la inclusión y exclusión está determinada por el estatus, la distribución de recursos, el poder que afecta a diferentes grupos marginados. La igualdad basada en derechos no da respuesta a esas desigualdades estructurales (Abasolo y Montero, 2012).

Figura 3. Gráfico concepto de igualdad.

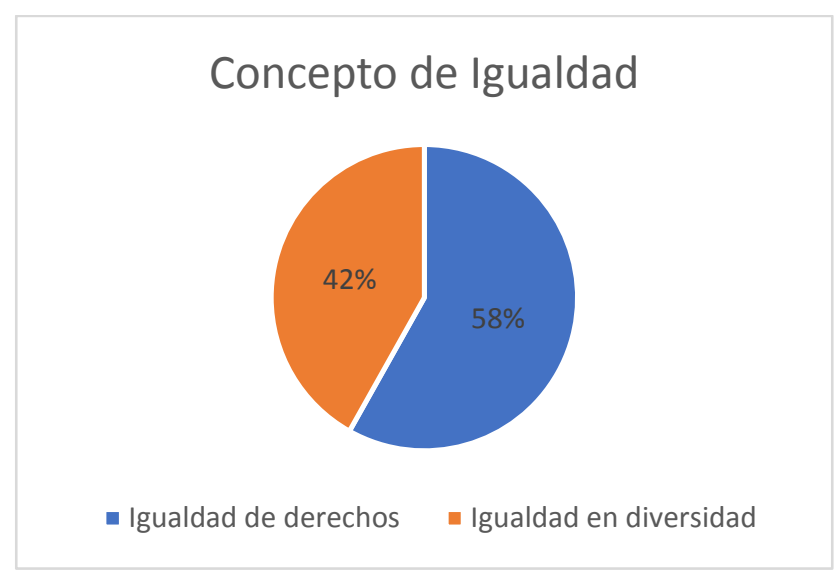

Fuente: Elaboración propia a partir del análisis de los PFC escogidos.

El $42 \%$ de las menciones entienden este principio como igualdad en diversidad, es decir, que toma en cuenta las diferencias. Esto se puede apreciar en frases como: "La escuela sea 
un lugar propicio para integrar las diversas necesidades de las estudiantes; recogiendo y valorando sus diferencias en cuanto a estilo y ritmos de aprendizajes, experiencias de vida, historia y composición familiar, origen social, gustos" (Escuela ${ }^{\circ}{ }^{2}$ ). Esta concepción de la igualdad tiene en su centro la diversidad y la inclusión de todas las personas, respetando y valorando aquello que las hace diferentes. Esta es la noción que se debe promover desde el feminismo pues conduce a sociedades más inclusivas y permite luchar contra todo tipo de exclusión social. Al mismo tiempo, la concepción de igualdad en diversidad plantea la importancia de reconocimiento de derechos diferenciados de grupos, como por ejemplo los derechos sexuales y reproductivos, trascendentales para el reconocimiento de problemáticas que afectan a mujeres, homosexuales, disidencias sexuales, entre otras personas. De ahí que resulte útil incorporar el concepto de ciudadanía sexual y reproductiva (Arnot, 2009).

\subsubsection{Valores}

La sociedad patriarcal se ha sustentado en una jerarquización de valores, en donde los masculinos asociados a la eficiencia, competencia, ambición de poder, el éxito, han prevalecido por sobre aquellos ligados a lo femenino relacionados con las vivencias, las relaciones, los deseos, etc. (Donoso, 2018). Por lo que, un nuevo modelo de ciudadanía debe sustentarse también en la reflexión y transformación del sistema de valores.

Como se puede apreciar en la Figura 4, las escuelas le dan un gran énfasis al desarrollo valórico. Los valores con mayor presencia son los explicitados en la Ley como diversidad, tolerancia, transparencia y probidad y otros no declarados en la Ley como el respeto, diálogo, solidaridad, responsabilidad. Sin embargo, la presencia de estos valores es más declarativa que real, puesto que tienen poco asidero en actividades prácticas que ayuden al estudiantado a desarrollarlos.

Figura 4: Gráfico presencia valores

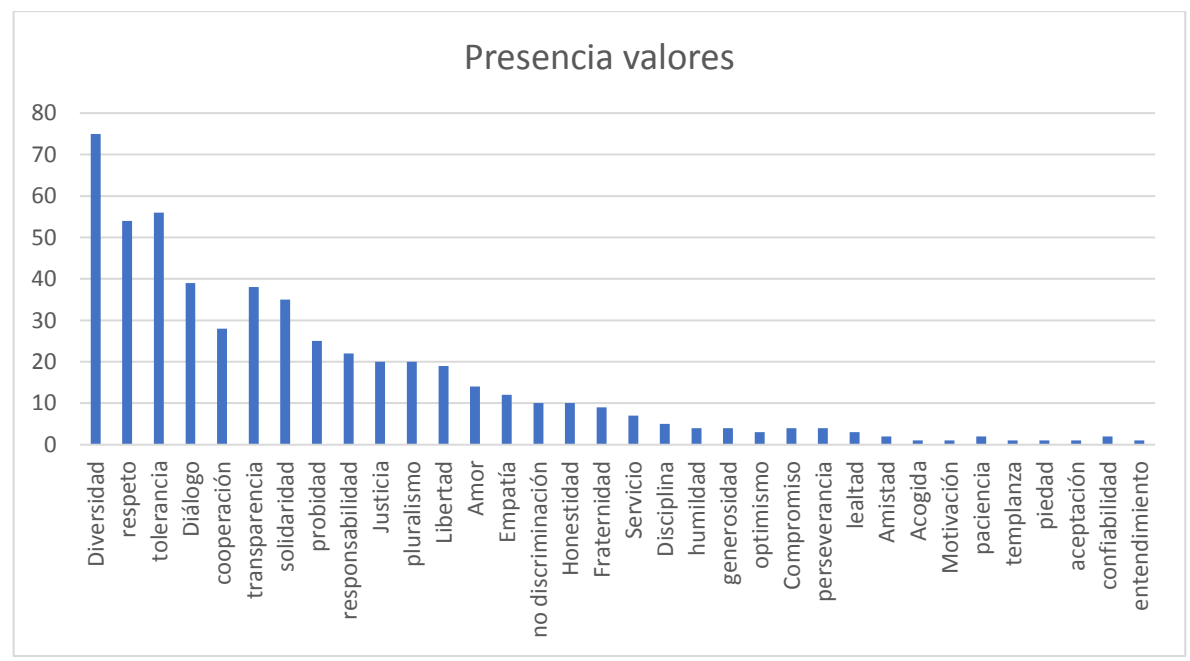

Fuente: Elaboración propia a partir del análisis de los PFC escogidos. 
Los valores identificados en los PFC se pueden relacionar con los valores propios de la escuela coeducadora en tres sentidos. El primero, que se ha denominado "valores propios de la coeducación", se refiere a aquellos que permiten la inclusión de todos los colectivos sociales. Para efectos de esta investigación se incluyeron en este grupo: la diversidad, el respeto, la cooperación, la empatía, la solidaridad y la generosidad. La escuela coeducadora también sostiene que es fundamental que se rescaten y potencien "los valores tradicionalmente femeninos", puesto que son estos los que han permitido el sustento de la vida humana. En los PFC se han encontrado valores como la humildad, el amor, el servicio, la acogida, la responsabilidad, la templanza y la piedad. Por último, para transformar el sistema de valores existentes, también es importante rescatar "valores que permitan luchar contra toda desigualdad" y les permitan a las y los estudiantes adquirir una actitud crítica en su vida cotidiana. En los PFC se encontraron valores como la no discriminación, la libertad, la justicia y el compromiso.

Figura 5. Gráfico presencia valores coeducación.

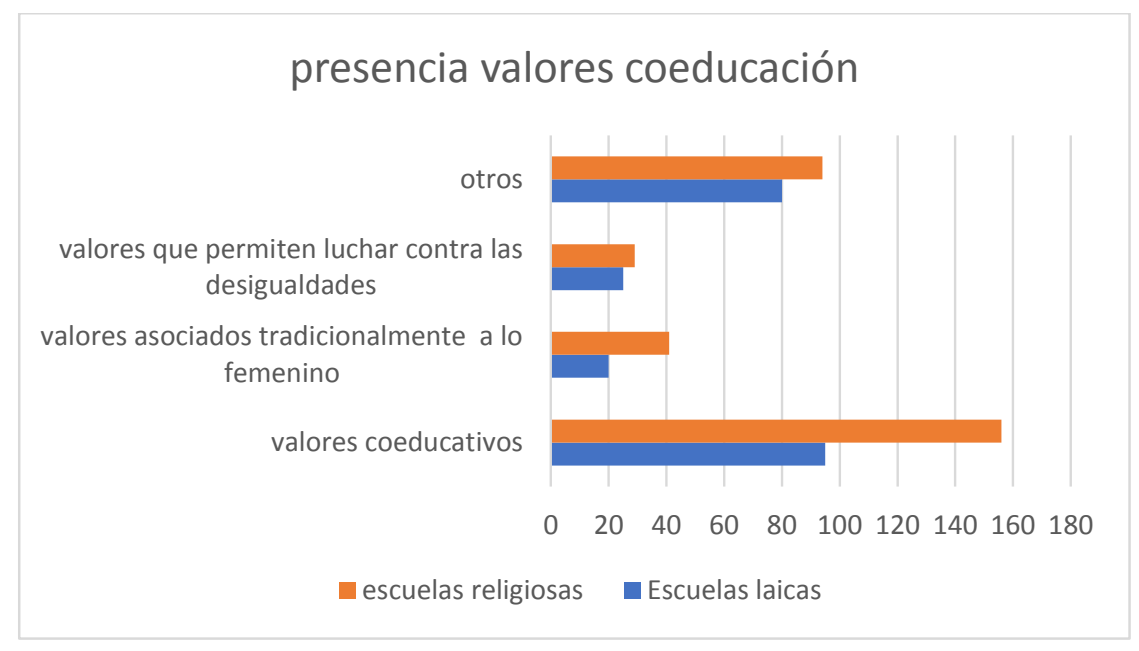

Fuente: Elaboración propia a partir del análisis de los PFC escogidos.

Como se puede apreciar en la figura 5, en las veinte escuelas analizadas existe una presencia importante de aquellos valores que permiten la coeducación, desde esta perspectiva se abren posibilidades para trabajar la perspectiva feminista en la formación ciudadana. Sin embargo, los valores asociados tradicionalmente al ámbito femenino y los que permiten luchar contra las desigualdades tienen escasa presencia.

\section{2. ¿CÓMO SE INCORPORA LA PERSPECTIVA FEMINISTA?}

En este apartado se busca identificar y reflexionar sobre cómo se trabaja la perspectiva feminista en los PFC. Para ello se identificaron las categorías de: metodologías utilizadas 
y temáticas que permiten incorporar la perspectiva feminista a la reflexión sobre ciudadanía.

\subsubsection{Metodologías}

Las metodologías corresponden al conjunto de técnicas de enseñanza que utiliza el profesorado con el objetivo de lograr determinados objetivos de aprendizaje. Mediante el "cómo" las y los docentes también transmiten pensamientos, ideas, valores. Como se aprecia en la figura 6 , se desarrollan principalmente aquellas metodologías consideradas participativas por sobre las tradicionales.

Figura 6. Gráfico metodologías utilizadas.

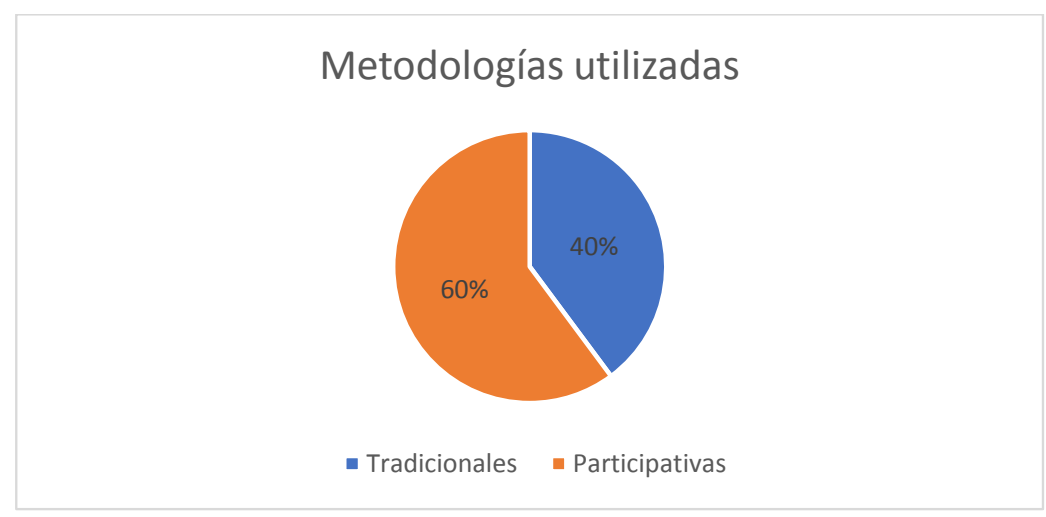

Fuente: Elaboración propia a partir del análisis de los PFC escogidos.

En relación a metodologías tradicionales, las escuelas desarrollan actividades como confeccionar ficheros informativos, visitas a instituciones del Estado, efemérides del día, actos cívicos, clases expositivas, desfiles, charlas, etc. Como, por ejemplo, "Efemérides del día. Cada profesor y profesora, de manera permanente y clase a clase, deberá escribir, luego de la fecha y antes del objetivo, la efeméride del día" (Colegio n²0). Este tipo de actividades se relacionan con formas de enseñanza que ponen el acento en lo que el/la docente o escuela transmite, mientras que los/las estudiantes son receptores de lo enseñado. La contribución de este tipo de actividades es muy baja en relación a ciudadanas y ciudadanos con un alto nivel de implicación y responsabilidad, necesarios para un modelo de ciudadanía en perspectiva feminista.

En relación a las metodologías participativas, en los PFC revisados se encontraron actividades cooperativas y no cooperativas, destacando mayoritariamente aquellas actividades no cooperativas como se puede apreciar en la figura 7. 
Figura 7. Gráfico frecuencia de aparición de actividades cooperativas vs no cooperativas en los PFC

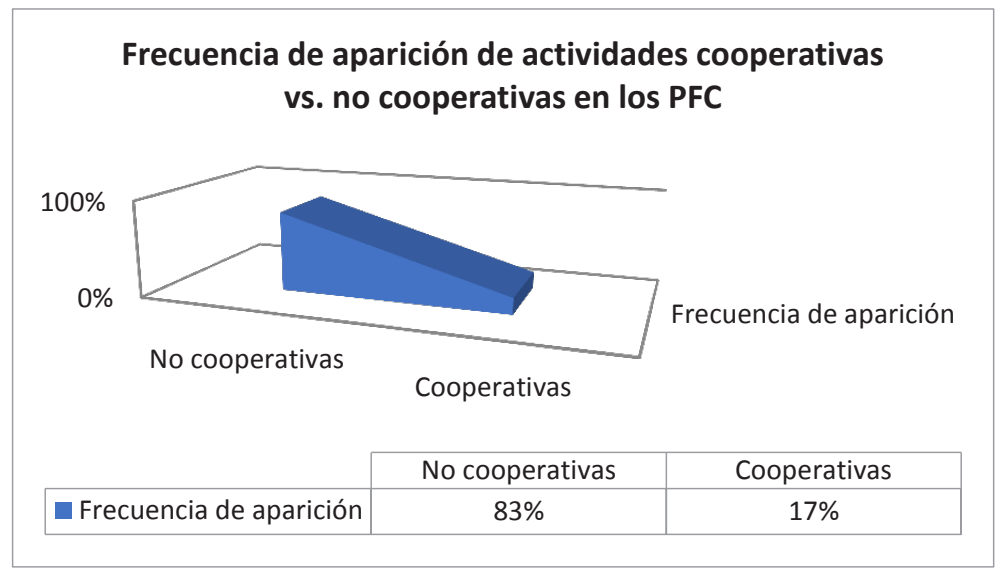

Fuente: Elaboración propia a partir del análisis de los PFC escogidos.

Dentro de las metodologías "participativas no cooperativas", se desarrollan actividades como la participación en los consejos de curso, torneos de debate, votación y conformación de Centro de Estudiantes, muestras culturales de otras nacionalidades, grupos de periodismo, entre otras. Si bien estas actividades implican la participación activa del estudiantado, son actividades episódicas y aisladas, que tienen el "riesgo de hacer de la formación ciudadana una experiencia que opera como hito, que no se articula con la enseñanza que tiene lugar en las asignaturas" (PNUD, 2018, p. 4). Por otra parte, la mayoría de estas actividades posiblemente implican la participación de un grupo estudiantes que suelen ser los más activos, con mayor liderazgo y compromiso con el establecimiento educativo.

Las metodologías participativas cooperativas, tienen una presencia mucho menor. Corresponden a aquellas donde el estudiantado participa activamente de los aprendizajes, mediante la colaboración entre distintos/as pares. Como se aprecia en el siguiente extracto: "Diseñar e incentivar prácticas pedagógicas que fomenten el análisis, la resolución de problemas, el trabajo en equipo y el pensamiento reflexivo en relación a la formación ciudadana" (Escuela $n^{\circ} 2$ ). Dentro de esta subcategoría se destacan acciones como la metodología de aprendizaje basada en proyectos, experiencias de reciclaje, actividades de voluntariado, entre otros. Este tipo de actividades a diferencia del anterior, implican la participación activa de todo el estudiantado.

\subsubsection{Temáticas que permiten incorporar la perspectiva feminista a la ciudadanía}

Esta categoría se compone de temas de debate actual que desde el movimiento feminista se han relevado como fundamentales en la reflexión y discusión política. Por esta razón, resultan esenciales para la formación ciudadana. Los temas pertenecientes a esta categoría tienen escasa presencia y aparecen de forma aislada en los PFC. De hecho, de las veinte escuelas revisadas existen siete en las que no existe ninguna referencia al respecto. 
En relación a los PFC donde estos temas tienen presencia, la mayor parte de ellas se presentan en la fundamentación o a modo de ejemplo y son muy pocas las actividades prácticas que se relacionan con esta categoría. Por ejemplo, en el siguiente PFC se señala que "se reserva de forma permanente un espacio del diario mural para la mantención de un fichero ciudadano, que comunique información de interés público, actividades del colegio relacionadas con formación ciudadana y temáticas de derechos, interculturalidad, estereotipos de género, diversidad" (Escuela n ${ }^{\circ}$ ). En este caso, el tema estereotipos de género, aparece como un ejemplo más entre los que se podrían desarrollar en el fichero ciudadano, pero no necesariamente indica que se va a trabajar el tema. Es decir, el tema está integrado en el PFC de manera ambigua.

Como se puede apreciar en la figura $n^{\circ} 8$, la subcategoría que más se menciona es la de diversidad sexual y de género. Esta subcategoría aparece principalmente en los fundamentos de los PFC, como se aprecia en el siguiente extracto: "como una forma de vida en la que se desarrolla la libre discusión de ideas y la existencia de diversas manifestaciones ideológicas, sexuales, raciales, religiosas" (Escuela $\left.\mathrm{n}^{\circ} 1\right)$.

Figura 8. Gráfico temáticas que permiten incorporar la perspectiva feminista a la ciudadanía.

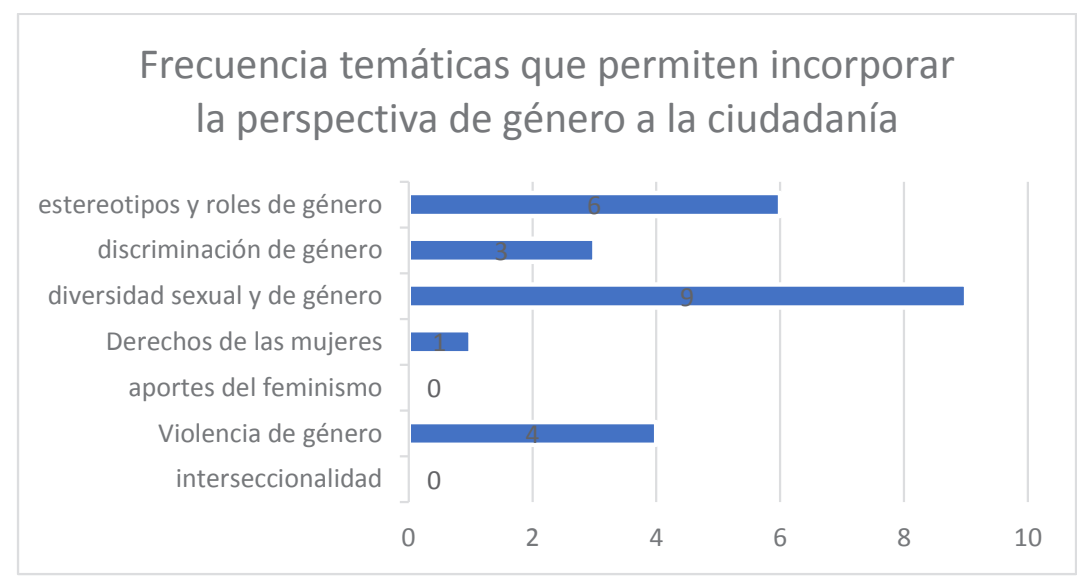

Fuente: Elaboración propia a partir del análisis de los PFC escogidos.

La segunda subcategoría que tiene mayor presencia es la de estereotipos o roles de género, la que se incorpora con el objetivo de superar estos estereotipos. Esta subcategoría tiene presencia tanto en la fundamentación como en el desarrollo de actividades. Esto se aprecia en el PFC que señala "Clases de orientación: reflexión crítica sobre estereotipos de género" (Escuela $\left.n^{\circ} 4\right)$.

La subcategoría derechos de las mujeres, tiene una sola aparición en los PFC analizados, mientras que las subcategorías interseccionalidad y aportes del feminismo, no tienen presencia, lo que puede evidenciar la escasa valoración o el desconocimiento que se le da a los aportes del feminismo en la construcción de la ciudadanía. 


\subsection{POSIBILIDADES Y LIMITACIONES QUE OFRECE LA LEY 20911 PARA INCORPORAR LA PERSPECTIVA FEMINISTA A LA CIUDADANÍA}

Luego de la revisión de veinte PFC se pueden indicar algunas posibilidades y limitaciones que ofrece la Ley 20911 para desarrollar una formación ciudadana en perspectiva feminista.

En primer lugar, se puede destacar que la Ley 20.911 tiene como propósito entender la ciudadanía como parte de la experiencia escolar. Es decir, que las y los estudiantes puedan implicarse y participar mediante las acciones establecidas por cada escuela en su PFC. Esto se refleja en que la subcategoría ciudadanía participativa, tiene una presencia bastante relevante. Una ciudadanía en perspectiva feminista debiese al menos permitir la inclusión de todos los colectivos sociales y para ello es fundamental incentivar la participación.

La Ley establece que en las escuelas se debe "promover el conocimiento, comprensión y análisis del Estado de Derecho y de la institucionalidad local, regional y nacional, y la formación de virtudes cívicas en los estudiantes" (Ley 20911, 2016), esto se traduce en que se da énfasis al conocimiento y comprensión de la institucionalidad política, sin existir un juicio crítico sobre sus deficiencias. Junto con esto, muchas de las actividades que implican la participación del estudiantado, se relacionan con el voto. En términos generales podría afirmarse que se fomenta una ciudadanía responsable, o de baja intensidad, que tiende a reproducir las estructuras sociales.

Se puede destacar la relevancia que se da en los PFC al desarrollo valórico. Esto permite fomentar una serie de valores que son propios de la coeducación, como diversidad, diálogo, solidaridad, cooperación, etc. En especial en la Ley se explícita la valoración de la diversidad social y cultural, que es uno de los valores centrales de la coeducación, puesto que permite rescatar, respetar y valorar las diferencias.

Para desarrollar el objetivo, "fomentar en los estudiantes la tolerancia y el pluralismo", el documento "Orientaciones para la elaboración del Plan de Formación Ciudadana" establece de forma explícita, que se pueden incorporar prácticas que fomenten la igualdad de género en la convivencia cotidiana (MINEDUC, 2016). Sin embargo, esta indicación no es una exigencia, por lo que se le otorga poca relevancia. De hecho, en ninguno de los PFC analizados se aprecia una acción concreta para fomentar la igualdad de género.

La Ley no establece ningún objetivo en que se promueva la igualdad. Esto se traduce en que en los PFC no se le da relevancia a este principio. La igualdad en diversidad debiese ser la base de todo modelo de ciudadanía que pretenda ser inclusivo y valore a todos los colectivos sociales.

El objetivo de la Ley "fomentar la participación de los estudiantes en temas de interés público" (Ley 20911, 2016), permite la incorporación de temas actuales en relación al género, como diversidad sexual y de género, violencia de género, interseccionalidad, entre otros. Estos temas pueden ser del interés del alumnado, se encuentran en el debate público y su reflexión permite el desarrollo de competencias ciudadanas. Sin embargo, queda en manos de los colegios incluir o no temas relacionados con las demandas feministas. Como se pudo apreciar en la revisión de los PFC seleccionados, estos temas quedan bastante relegados.

La Ley no se refiere a las metodologías que pueden utilizarse para confeccionar los PFC, por lo que queda en manos de cada escuela cómo hacerlo. Esto por un lado es positivo, porque permite desarrollar la creatividad y realizar acciones que tengan que ver con la identidad de cada escuela. Pero en la práctica, se desarrollan metodologías, que, aunque 
son participativas, suelen estar poco vinculadas a las actividades de enseñanza-aprendizaje del aula y/o incluyen a grupos de estudiantes puntuales.

\subsection{ORIENTACIONES PARA TRABAJAR UNA CIUDADANÍA EN PERSPECTIVA FEMINISTA}

En este apartado, se sintetizan algunas orientaciones que permitirían incorporar la perspectiva feminista en la formación ciudadana. Estas se han establecido a partir de la revisión teórica y de los resultados obtenidos del análisis de los PFC, por lo que pretenden ajustarse a la realidad educativa chilena.

Con respecto a los contenidos que deben estar presentes en los PFC, en primer lugar, como sugieren Cobo (2008) y Abasolo y Montero (2012) se debe abordar aquellos conceptos políticos relacionados con la ciudadanía como: Derechos Humanos, igualdad, Democracia, institucionalidad política, pero desde una perspectiva crítica. Es decir, las y los estudiantes deben discutir, reflexionar y sobre todo cuestionar las exclusiones e injusticias que se generan en el sistema político actual tanto nacional como global. Para ello es importante rescatar las herramientas teóricas y metodológicas de la teoría feminista. De esta manera se puede desarrollar una cultura de cuestionamiento a la realidad y al mismo tiempo transmitir a las niñas y jóvenes que las mujeres y otros colectivos oprimidos también son actores sociales y políticos con capacidad de agencia (Arnot, 2009).

Uno de los contenidos claves que se puede integrar a los PFC es la reflexión y cuestionamiento de los estereotipos sexistas (Abasolo y Montero, 2012). Esto se puede hacer mediante la incorporación de diferentes temas de debate actual, en los que el estudiantado pueda analizar los estereotipos en plataformas como videos con alta reproducción en redes sociales, prensa en diferentes formatos, publicidad, libros, música, cine, etc. Por otra parte, las y los estudiantes pueden reflexionar sobre los estereotipos sexistas presentes en su vida cotidiana a nivel de sus relaciones sociales, familiares, escolares y sexuales a partir de las que se pueden discutir temas relevantes como: la división sexual del trabajo, las identidades de género y sexuales y la repartición de las labores domésticas y del cuidado.

Mediante la incorporación de diferentes modelos o referentes de ciudadanía, se puede romper con los estereotipos y ofrecer mayor visibilidad a todos los colectivos sociales. En este sentido Massip y Castelví (2019), señalan la importancia de la perspectiva interseccional en la elección de las y los protagonistas que forman parte de los relatos. Para estos, la perspectiva interseccional permite visibilizar y dar respuesta a las diversidades y al mismo tiempo explicarlas en relación al poder, dominación y exclusión. Una de las acciones más utilizadas por los PFC analizados son las efemérides del día, a través de esta acción se pueden relevar las acciones realizadas por feministas, personas que luchan por la paz, representantes de colectivos LGBTIQ, indígenas, ecologistas, etc. Su rescate permite demostrar la capacidad de agencia de todo tipo de personas. Estos protagonistas de la ciudadanía, además, pueden reemplazar a los tradicionales próceres de la patria asociados a conflictos bélicos.

Asimismo, es importante incluir a la reflexión ciudadana aquellos temas que tradicionalmente se han excluido por pertenecer a la esfera privada (Arnot, 2009). Se debe cuestionar "el legado histórico que marginó la esfera de la vida familiar cotidiana y por supuesto la maternidad y virtudes femeninas, de los análisis de los derechos, los deberes, la justicia y la libertad" (Subirats, 2009, p. 11). Al entender que lo personal es político, la formación ciudadana puede incluir temas como como la afectividad y la sexualidad, 
la violencia de género, los cambios sociales de la vida familiar, la diversidad sexual y de género, etc.

Es esencial también acompañar las reflexiones políticas de un desarrollo valórico, asentado en los principios de la coeducación. El foco de los valores debe estar en la diversidad que permite la aceptación e inclusión de todos los colectivos sociales (Roset et al., 2008). Junto con esto se debe desarrollar una base ética que tenga como centro la indignación respecto a la injusticia (Arnot, 2009). Para tales efectos, es posible incluir el estudio de una serie de movimiento sociales contrahegemónicos tanto históricos como actuales, como el feminismo, el ecologismo, el movimiento estudiantil, movimientos por los Derechos Humanos, movimiento LGTIBQ, entre otros; que permitan desarrollar referentes valóricos múltiples y diversos, que tienen en común la lucha contra las injusticias sociales.

Es fundamental, además, que se utilicen metodologías que permitan el desarrollo del pensamiento crítico. Las y los estudiantes deben ser capaces de hacer un juicio crítico a las estructuras políticas, sociales, culturales, económicas, que provocan la exclusión social. El pensamiento crítico es fundamental, para visibilizar las estructuras patriarcales que permean de manera cotidiana la escuela, la familia, las relaciones sociales, el trabajo, la política, etc. En ese sentido, Arnot (2009) propone desarrollar una pedagogía crítica de la diferencia, es decir, una pedagogía que combine la tradición de las pedagogías críticas con los postulados de la diferencia, permitiendo que las y los estudiantes comprendan los discursos de género y se posicionen de manera crítica frente a la asignación de estereotipos y al sistema patriarcal.

\section{CONCLUSIONES}

Este artículo tuvo como primer objetivo analizar el modelo de ciudadanía que ofrecen los PFC desde una perspectiva feminista, considerando el tipo de ciudadanía que trabajan, los valores que transmiten, y la visión de igualdad que promueven. Los resultados obtenidos permiten señalar que los PFC mayoritariamente se han elaborado desde la perspectiva del ciudadano, y no han incluido las aportaciones que el feminismo ha hecho en la materia, excluyendo de esta manara a la diversidad de experiencias y realidades. Esto se puede reflejar en la relevancia que tiene la subcategoría ciudadanía responsable y el escaso desarrollo que tiene la ciudadanía orientada a la justicia. Además de la escasa presencia del principio de igualdad, fundamental para toda reflexión que pretenda incluir injusticias sociales.

En el segundo objetivo se buscaba identificar temáticas y metodologías que permiten trabajar desde una perspectiva feminista. De acuerdo con las evidencias encontradas es posible afirmar que la mayoría de las metodologías utilizadas son participativas. Sin embargo, la mayor parte de ellas no son cooperativas, por lo que dificultan la inclusión de toda la comunidad educativa. Por otra parte, los temas de debate actual que permitirían incorporar la perspectiva feminista a la formación ciudadana tienen escasa presencia.

A partir del análisis realizado se puede responder la pregunta que dio origen a esta investigación: ¿Qué posibilidades ofrece la Ley 20911 para desarrollar una ciudadanía que incorpore la perspectiva feminista? Al respecto puede indicarse que la Ley otorga escasas posibilidades para incorporar esta perspectiva, debido a que: prioriza la comprensión de la institucionalidad política sin hacer un juicio crítico de ella; no se incorporan de forma 
explícita temas de interés público que ha relevado el feminismo en los últimos años; no existe un posicionamiento frente a los estereotipos de género; y se le da escasa relevancia al principio de igualdad.

Finalmente, este estudio pretendía aproximarse a evidenciar como se está educando en ciudadanía a niños, niñas y juventud chilena desde una mirada feminista. Esta investigación es una invitación a reflexionar sobre las implicancias que tiene prescindir de esta perspectiva. Al mismo tiempo puede ser la base para tomar decisiones que permitan hacer de la educación para la ciudadanía un área que posibilite la lucha contra las desigualdades sociales y fomente la democracia desde las escuelas. Para ello, se pueden tener en cuenta los siguientes aspectos:

a) Abordar conceptos de la teoría política desde una perspectiva crítica, utilizando herramientas teóricas del feminismo.

b) Reflexionar y cuestionar la permanencia de estereotipos sexistas.

c) Incluir a la reflexión ciudadana temas que tradicionalmente se han relacionado con el mundo privado, pero que tienen una relevancia política.

d) Desarrollar metodologías que permitan la inclusión de todas y todos los estudiantes.

e) Incorporar metodologías que potencien el pensamiento crítico.

f) Impulsar valores de la coeducación y desarrollar una ética que tenga como base la indignación frente a las injusticias.

Las características con las que se presenta la Formación Ciudadana en la actualidad implican una ciudadanía que reproduce el sistema político imperante y al mismo tiempo las desigualdades sociales. Sin embargo, la incorporación de elementos de la teoría feminista representa una alternativa real que desafía a la actual forma de educar en ciudadanía, con el objetivo de construir una ciudadanía que busque la transformación del sistema patriarcal y las injusticias sociales.

\section{REFERENCIAS BIBLIOGRÁFICAS}

Abasolo, O. y Montero, J. (2012). Guía Didáctica de Ciudadanía con perspectiva de género. Igualdad en diversidad. Para profesorado de segunda etapa de Eso y Bachillerato. FUHEM Ecosocial. https://www.inmujeres.gob.es/areasTematicas/educacion/publicaciones/docs/guia_ didactica_ciudadania_FUHEM.pdf

Agencia de Calidad de la educación. (s/f). Desarrollo personal y social: otros indicadores de calidad educacional. MINEDUC.

Aguiló, A. (2009). Ciudadanía como proceso de emancipación: retos para el ejercicio deciudadanías de alta intensidad. Astrolabio, (9), 13-24. https://www.raco.cat/index.php/Astrolabio/article/ view/197698

Arnot, M. (2009). Coeducando para una ciudadanía en igualdad. Morata.

Burgos, E. (2010). Identidades de género: una mirada feminista. En R. Aquezar y R. Rodríguez. Educación cívica. Democracia y cuestiones de género (pp. 243-272). Icaria.

Cabrera, F. (2002). ¿Qué educación para que ciudadanía? En E. Soriano. Interculturalidad: Fundamentos, programas y evaluación (pp. 83-129). La Muralla.

Cabrera, F. (2011). Técnicas e instrumentos de evaluación, una propuesta de clasificación. Revista d' innovació i reserca en Educació, 4(2), 112-124. DOI:10.1344/reire2011.4.2428 
Estudios Pedagógicos XLVII N 3: 99-123, 2021

FORMANDO EN CIUDADANÍA A NIÑAS Y NIÑOS. PERSPECTIVA FEMINISTA EN LOS PLANES DE FORMACIÓN CIUDADANA (PFC) DE LAS ESCUELAS CHILENAS

Cobo, R. (1995). Género. En C. Amoros. 10 palábras sobre mujer. Verbo divino. . (2008). Educar en la ciudadanía. Perspectivas feministas. Catarata.

Corbetta, P. (2007). Metodologías y técnicas de investigación social. Mc Graw Hill.

Donoso, T. (2018). Perspectiva de género en la universidad como motor de innovación. En A. Rebolledo-Catalán, E. Ruz-Pinto y L. Vega-Caro. La universidad en clave de género (pp. 2352). Octaedro.

Facio, A. (2005). Feminismo, género y patriarcado. Revista sobre enseñanza del derecho en Buenos Aires, 259-294. http://repositorio.ciem.ucr.ac.cr/jspui/handle/123456789/122

Guerrero, E., Hurtado, V., Azúa, X. y Provoste, P. (2011). Material de apoyo con perspectiva de género para formadores y formadoras. Hexagrama consultoras. https://bibliotecadigital. mineduc.cl/bitstream/handle/20.500.12365/14919/curriculo-de-genero-oculto-para-profesorases.pdf? sequence $=1 \&$ isAllowed $=\mathrm{y}$

Hanish, C. (2016). Lo personal es político. Ediciones feministas lúcidas. http://autonomiafeminista. cl/wp-content/uploads/2016/07/lo-personal-es-pol\%C3\%ADtico_lucidas.pdf

Ley 20911 de 2016. Crea el Plan de Formación Ciudadana para los establecimientos educacionales reconocidos por el Estado. 02 de abril de 2016. www.leychile.cl

Massip, M. y Castellví, J. (2019). Poder y diversidad. Los aportes de la Interseccionalidad a la didáctica de las ciencias sociales. CLIO. History and History teaching, 139-154. https://dialnet. unirioja.es/servlet/articulo?codigo $=7351991$

MINEDUC. (2016). Orientaciones para la elaboración del Plan de Formación Ciudadana. Ministerio de Educación. https://www.mineduc.cl/wp-content/uploads/sites/19/2016/04/ OrientacionesPFC.pdf

Mouffe, C. (1999). El retorno de lo político. Comunidad, ciudadanía, pluralismo, democracia radical. Paidos.

Ochman, M. (2006). En busca de una nueva sociedad. Los aportes de la teoría feminista a la reformulación del mundo moderno. Desafíos, (15), 371-387. https://www.redalyc.org/articulo. oa? id=359633160011

Pagès, J. y Santisteban, A. (2007). La educación para la ciudadanía hoy. En Educación para la ciudadanía. Wolters Kluwer.

Pateman, C. (1988). El contrato sexual. Antrophos.

PNUD. (2015). Desarrollo Humano, tiempos de politización. Programa de las Naciones Unidas para el Desarrollo. https://www.cl.undp.org/content/chile/es/home/library/human_development/lostiempos-de-la-politizacion.html

(2018). Estudio sobre la puesta en marcha del Plan de Formación Ciudadana. Programa de las Naciones Unidas para el Desarrollo. http://www.ciudadaniayescuela.cl/wp-content/ uploads/2018/04/Estudio-puesta-en-marcha-del-Plan-de-Formaci\%C3\%B3n-Ciudadana.pdf

Roset, M., Pagés, E., Lojo, M. y Cortada, E. (2008). Guía de coeducació per als centres educatius. Pautes de reflexió, recursos per a l'elaboració d'un projecte de centre. Institut Catalá de la Dona.

Sabariego, M. (2016). La investigación educativa: génesis, evolución y características. En R. Bisquerra. Metodología de la investigación educativa (pp. 49-85). La muralla.

Sánchez, A. e Iglesias, A. (2008). Currículum oculto en el aula: estereotipos en acción. En R. Cobo. Educar en la ciudadanía. Perspectivas feministas (pp. 123-150). Catarata.

Sau, V. (1990). Diccionario ideológico feminista, Vol 1. Icària.

Stolcke. V. (2004). La mujer es puro cuento: la cultura del género. Estudios feministas, florianópolis, 12(25), 77-105. https://doi.org/10.1590/S0104-026X2004000200005.

Subirats, J. y Parés, M. (2014). Cambios sociales y estructuras de poder ¿Nuevas ciudades, nueva ciudadanía? Interdisciplina, (2), 97-118. Doi: http://dx.doi.org/10.22201/ ceiich.24485705e.2014.2.46526

Subirats, M. (2009). Prólogo a la edición española. En M. Arnot, Coeducando para una ciudadanía en igualdad (pp. 9-16). Morata. 
Troncoso, L., Follegati, L. y Stutzin, V. (2019). Más allá de una educación no sexista: aportes de pedagogías interseccionales. Pensamiento educativo. Revista de investigación educativa latinoamericana, 56(1), 1-15. DOI: https://doi.org/10.7764/PEL.56.1.2019.1

Varela, N. (2018). Feminismo para principiantes. Biblioteca de bolsillo.

Westheimer, J. y Khane, J. (2002). Educating the good citizen. The politics of school-based civic education programs. Paper to be presented at the annual meeting of the American Political Science Association (APSA).

Young, I. (1990). La justicia y la política de la diferencia. Cátedra. 
